\title{
BLOCKCHAIN ENABLED IOTS TOWARDS ACHIEVING THE SUSTAINABLE DEVELOPMENT GOALS
}

Nasser Hassan Abosaq

Assistant Professor, Computer Science and Engineering Department. Yanbu University College, Yanbu Industrial City, (Kingdom of Saudi Arabia). E-mail: abosaqn@rcyci.edu.sa ORCID: https://orcid.org/0000-00031354-3170

\section{Citación sugerida:}

Abosaq, N. H. (2021). Blockchain enabled IoTs towards achieving the Sustainable Development Goals. 3C Tecnología. Glosas de innovación aplicadas a la pyme, Edición Especial, (mayo 2021), 23-33. https:// doi.org/10.17993/3ctecno.2021.specialissue7.23-33 


\section{ABSTRACT}

The Blockchain enabled Internet of Things (IoTs) has emerged as an impactful research domain in recent years because of its focused global interest owing to the United Nations' commitment to achieve 17 Sustainable Development Goals by the year 2030. The Blockchain enabled IoTs has also received considerable attention from industry and academia due to its vast ranged potential applications in many explored fields. The Blockchain enabled IoTs technology has crossed the boundaries of cryptocurrency infrastructure and has become an integrated and distributed Systems of Systems ( $\mathrm{SoS})$ networked technology. It is because of a paradigm shift from centralized to the devolved and from static to dynamic networks of networks that this technology has received a wide enthusiasm and attention from researchers. This research is focused on challenges and opportunities of Blockchain enabled IoTs on cloud infrastructure, $5 \mathrm{G} / 6 \mathrm{G}$ wireless systems and its computing issues for achieving United Nations' sustainable development goals by 2030 worldwide. As a use case scenario, we highlight the latest advancements in blockchain enabled IoTs, its various potential applications, future research directions and its infrastructure available in the research readings. Investigation into the important factors considered as technical challenges to the socio-economic development with respect to achieving sustainable development goals are also reviewed. Numerous case studies on Blockchain enabled IoTs in various application areas of the interest falling in SDGs domain have been performed the interpretive case study approach is selected to gather the data and its results, and a protocol is defined to design the structure.

\section{KEYWORDS}

Blockchain, IoT, 5G, 6G, Wireless technology, Sustainable development goals. 


\section{INTRODUCTION}

The Millennium Development Goals (MDGs) set forth by United Nations in 2016 were succeeded by the 17 Sustainable Development Goals (SDGs) targeted to be achieved by the year 2030 (United Nations, n.d. b). The SDGs are the targets for global wellness by easing the human lives through zero hunger, equitable quality education, sound environment, productive employment, economic growth through industrialization, green energy, global partnership, research and innovation on underwater resources, technology and many more. Emergence of 5G/6G universal telecommunication systems, 3D printing, blockchain, unmanned vehicles, drones as means of smart transportation and innovations in Artificial Intelligence (AI), Cloud computing, big data and data analytics are all set to aid in the UN's efforts for achieving SDGs by the year 2030. The world has entered a new era of technological development through fourth industrial revolution (4IR) of digital divide with many devastating challenges like COVID 19, environmental degradation, global warming, growing urbanization and natural disasters; all severely need multi-stakeholder partnerships among North-South, South-South and triangular regional and international digital connectivity. For coping with all these challenging global circumstances, nations are striving for innovative ideas and the Blockchain enabled IoTs is one of the promising technologies for designing robust digital networking responsible for connecting the humans, machines, and devices.

Internet of Things (IoTs) has integrated physical industrial operations with cyberspace technology in all its applications ranging from manufacturing to logistics to supply chain management. This has decentralized and diversified many industrial processes and systems and thus has created numerous process flow issues of interoperability of systems and devices, scalability, security, and privacy, trusted reliance and the most important one is the complex networking topologies due to Distributed Denial of Service (DDoS). The emergence of blockchain technology as a distributed system and its marriage with IoTs as a Service-oriented-Architecture have posed a sigh of relief for smooth industrial workflow of complex processes towards the successful business plans (Dorri et al., 2016). Because of the inherited property of being a comparatively secure and trusted system due to chained blocks data structures, immutability, and irrevocability, blockchain technology has received a sense of trustworthiness and confidentiality to execute guaranteed operations by all its 
potential end users. Hence adopting the blockchain technology for secure IoT applications has gained wide acceptance both in industry and researching academia (Ahmad \& Salah, 2018).

This research is focused on challenges and opportunities of Blockchain enabled IoTs on many industrial applications, cloud infrastructure and 5G/6G wireless systems for achieving United Nations' sustainable development goals by 2030 worldwide. As a use case scenario, we highlight the latest advancements in blockchain enabled IoTs, its various potential applications and future research directions. Investigation into the important factors considered as technical challenges to the socio-economic development with respect to achieving sustainable development goals are also reviewed.

\section{CHALLENGES AND OPPORTUNITIES OF BLOCKCHAIN ENABLED IOTS}

In Blockchain enabled IoTs security is realized as one of the main concerns by the researchers whereas the industrialized IoTs lack sufficient computing resources to tackle crypto-materialized protocols. There is an acute lack of common platform to deal with resource constrained low memory IoTs' ability to handle big data analytics challenge. Consequently, modifications in DDoS, smart contracts (Christidis \& Devetsikiotis, 2016), Service oriented layered Architecture, edge-IoT system, modification in authentication procedures to secure from external threats, multi-layered decentralization both in IoTs and Blockchain and many other enabling approaches have been proposed to overcome the challenges of blockchain-IoT nexus (Ahmad \& Salah, 2018).

However, the opportunities in this era outscore the present challenges. While perceiving the potential of technology in the context of achieving UN's sustainable development goals by 2030 , the enormous opportunities in blockchain enabled IoTs further drag us towards researching on multi-stakeholder economic growth, Machine-to-Machine networking, smart cities' automated transportation systems, innovative startups, biomedical and healthcare applications' sensors, cybersecurity, and supply chain management of all types and, last but not the least. the information and communication technologies. 


\subsection{ARCHITECTURAL REQUIREMENT OF BLOCKCHAIN-IOT}

Blockchain enabled IoT can be realized as an information system with a set of parameters to form a physical solution of any targeted prerequisites based upon axiomatic design theory. Such an axiomatic design system is an interdependent and interconnected mechanism of three entities, i.e., the customer's domain requirements (CRs), the functional requirements (FRs) and the physical solutions (PSs) as shown in Graphic 1 (Cochran et al., 2016). The axiomatic design theory is based upon mutual agreements that what customers desire to receive (FRs) is mapped to how those are produced (PSs). Hence there is a strong level of satisfaction between the involved parties as far as physical solutions are concerned.

What?

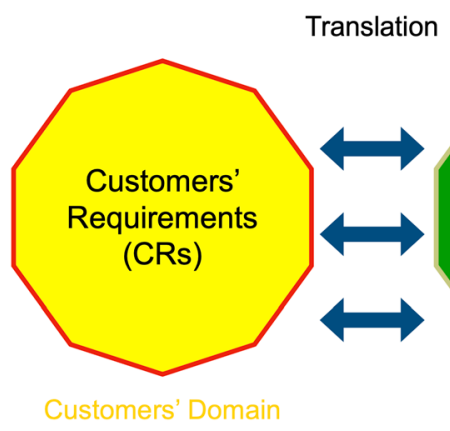

How?

Mapping

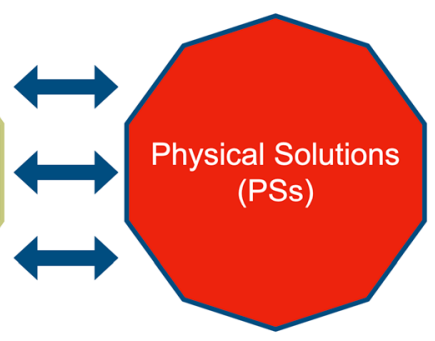

Physical Domain

Graphic 1. ADT for complex system design.

Source: (Cochran et al., 2016).

The IoT exhibits a non-static, dynamically oriented open environment where continuously changing services are involved requiring multi-vendor Quality of Service (QoS) responses to manage big data and its transmission load. Secondly the blockchain technology encompasses its inherent potential to deal with security threats due to Service oriented architecture of IoT as shown in Graphic 2. Other security threats like at Physical Layer (Interference, Insecure configuration, Spoofing and Physical Security), Network Layer Security (Like Resource Depletion, Identity and Authentication and Protocols) and Service Layer Security (like Insecure software, Single point of failure, Integrity and Privacy) can be well managed through deployment of blockchain infrastructure incorporating centralized and decentralized layers (Viriyasitavat et al., 2019). 


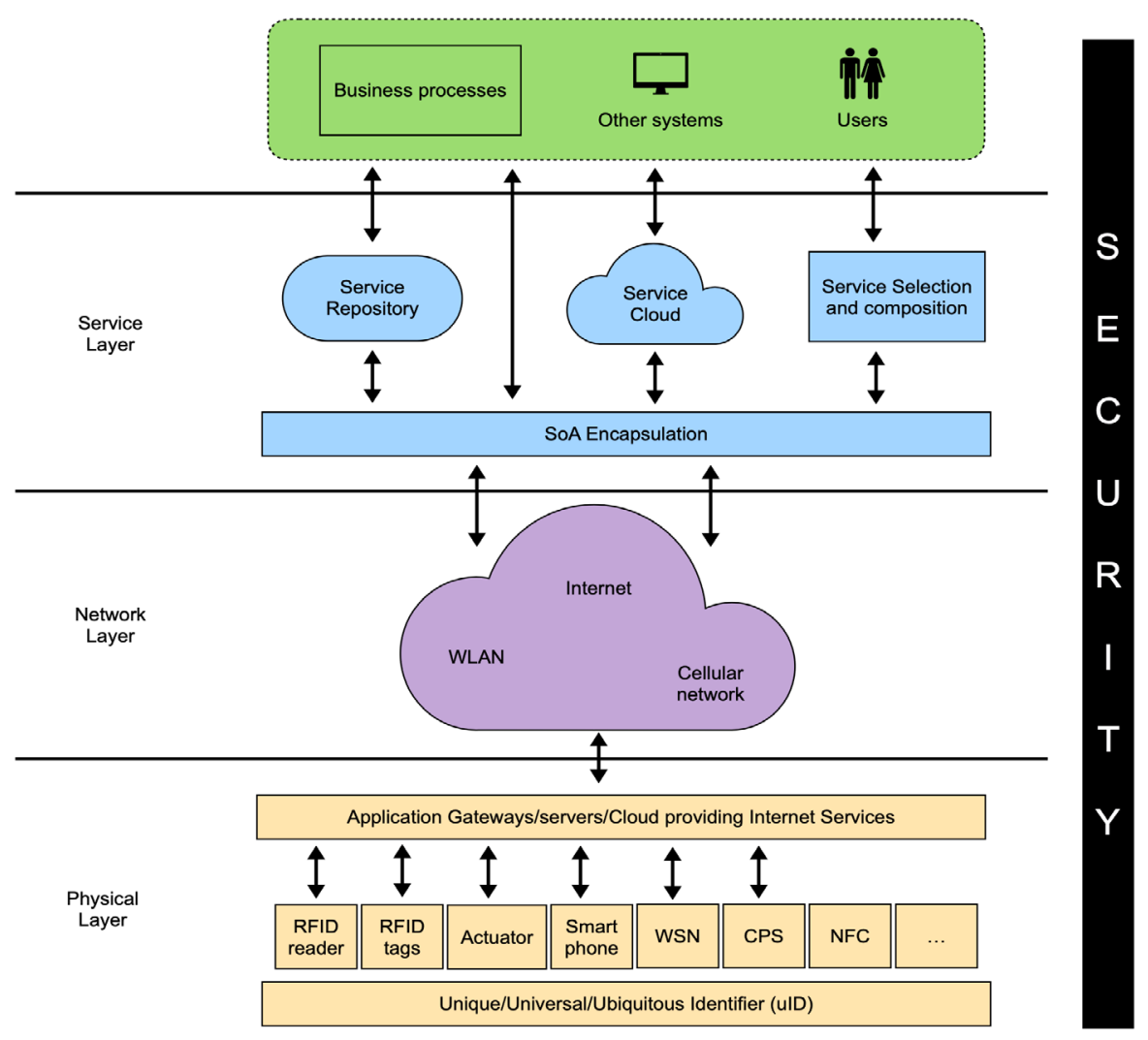

Graphic 2. SoA-based loT Architecture.

Source: (Viriyasitavat et al., 2019).

Additionally, access control protocols to safeguard IP spoofing in a distributed environment through smart contracts (Christidis \& Devetsikiotis, 2016) is also essential which can be managed via blockchain ledger. This has embarked upon a novel idea of inventing Public Biometric Infrastructure in the blockchain enabled IoT.

\subsection{POTENTIAL APPLICATIONS AND FUTURE RESEARCH DIRECTIONS}

Alexandru Stanciu is working on a novel standard called by him as Hyperledger Fabric for blockchain enabled IoTs under the platform of IEC 61499 standard (Viriyasitavat et al., 2019). This standard is inventing the development of standards for inputs, outputs, and necessary protocol operations for blockchain enabled IoTs. We are of the opinion that many of the UNs 17 SDGs can only be leveraged if technology is considered as priority option of the developing countries. The potential applications include widespread integration of contactless digital human computer interconnection with no mankind intervention. It is 
predicted that internet usage will be 28.5 billion devices by the year end 2022 means that per capita network connected devices will stand at 3.6 by 2022 .

Certainly, such a huge mobilization of network growth will emerge in generation of trillions of bytes of data in many human prioritized applications like healthcare, smart cities, environmental issues, GIS \& Remote sensing, agricultural and food security, intelligent transportation, green energy, and socio-economic technological growth. This all requires, among many other things, a systematic integration of blockchain to the industrial IoT for achieving the UNs SDGs by 2030.

\section{METHODOLOGY}

For validating the idea of nexus between blockchain and IoT, the many use case scenarios have been developed in the author's departments. This has facilitated tangible results to yield. For example, one use case scenario is e-networked business model consisting of student association, university administration and external and internal buyers to purchase any facilities owned by the university through advertisement mechanism. The transfer of access rights has been invoked between the involved parties or stakeholders.

The public and private blockchain benefits and disadvantages have been identified using different scenario conditions. A robust architecture is suggested comprising of different users, devices, networking resources, self-executable crypto contracts, storage repositories, auditable processes etc. An algorithm based on python file is created to act as a servicing program responsible for load balancing and leader election that provides link between the two blockchains. Our experimental setup is caried out on HP Notebook with 12 GB memory having core i7 processor. Etherum software 1.8.27 and python 3.7 programming language is used with Web3 library V 4.8.3.

Public and private nodes were designated. High-level language Solidity 0.5 is used for writing smart contracts (Christidis \& Devetsikiotis, 2016). Various decentralized and nondistributed options have also been studied to analyze scalability. The scenarios developed are for Access Generation (Pal et al., 2019), Electricity Cost as shown in Graphic 3 and 4 respectively, hostel accommodation cost, sweet water provision cost. 


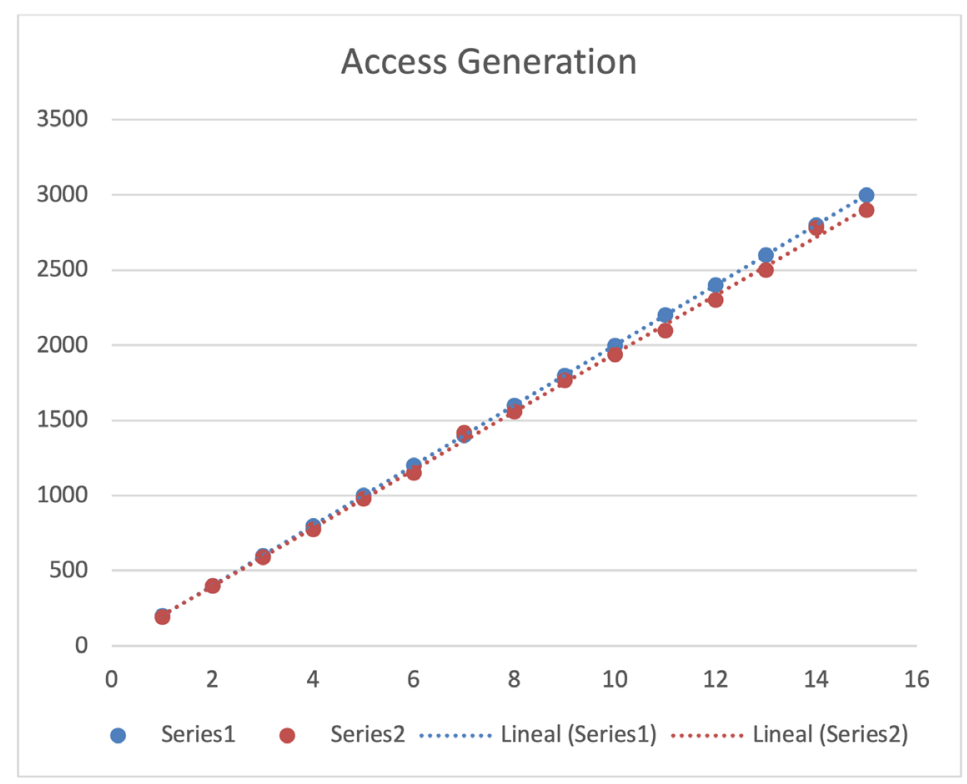

\section{Graphic 3. Access Generation.}

Source: own elaboration.

In furtherance to this experimental work, the author has executed many case studies on applications concerning blockchain enabled IoTs falling in SDGs domain.

\section{RESULTS}

For gathering fruitful results, the interpretive case study approach is used to gather the data. A protocol was defined to design the structure. Several interviews and brainstorm sessions were done in the author's university/ departments. 15 social domains were selected where blockchain enabled IoT data is gathered, transformed, and backed up using, ICT, AI, and Machine Learning Tools. To verify the delegation chain, two design options as serries 1 and series 2 were analyzed as shown in access generation graph using Breadth First Search algorithm to ensure the contract trail. The results show that waiting time is high enough. To reduce this waiting time, algorithm is improved in Electricity Cost as the cost has linear relationship with delegation depth as shown in Graphic 4. By introducing the novel algorithm, the blockchain efficacy has improved. 


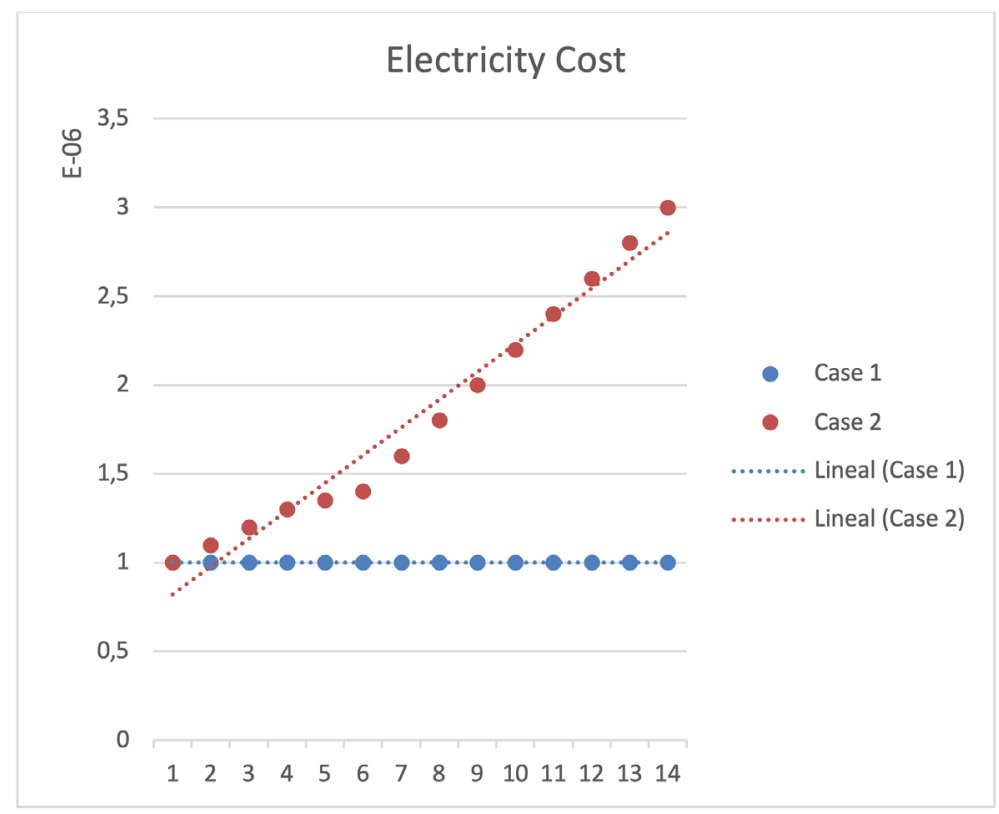

Graphic 4. Electricity Cost.

Source: own elaboration.

\section{CONCLUSIONS}

In this research, the author has considered the UN's 17 sustainable development goals as the motivation to use blockchain enabled IoTs as a candidate technology for achieving the goals by 2030. Having noticed the lack of security in block chain technology and resource constrained architecture of the industrialized IoTs to tackle crypto-materialized protocols, the emergence of private blockchain annexed with IoTs can contribute as a fullfledged secure system. This research shows that blockchain enabled IoTs can prove to be a promising technology towards achieving the stainability.

\section{ACKNOWLEDGMENT}

The author acknowledges the cooperation of Computer Science and Engineering Department and the management of Yanbu University College, Yanbu Industrial City, Kingdom of Saudi Arabia for supporting this research. 


\section{REFERENCES}

Ahmad, M., \& Salah, K. (2018). IoT security: Review, blockchain solutions, and open challenges. Future Generation Computer Systems, 82, 395-411. https://doi.org/10.1016/j. future.2017.11.022

Christidis, K., \& Devetsikiotis, M. (2016). Blockchains and Smart Contracts for the Internet of Things. IEEE Access, 4, 2292-2303. https://ieeexplore.ieee.org/ document/7467408

Cochran, D. S., Jafri, M. U., Ghu, A. K., \& Bi, Z. (2016). Incorporating design improvement with effective evaluation using the Manufacturing System Design Decomposition (MSDD). Fournal of Industrial Information Integration, 2, 65-74. https:// doi.org/10.1016/j.jii.2016.04.005

Dorri, A., Kanhere, S. S., \& Jurdak, R. (2016). Blockchain in internet of things: challenges and solutions. arXiv preprint arXiv:1608.05187. https://arxiv.org/abs/1608.05187

Pal, S., Rabehaja, T., Hill, A., Hitchens, M., \& Varadharajan, V. (2019). On the integration of blockchain to the internet of things for enabling access right delegation. IEEE Internet of Things fournal, 7(4), 2630-2639. https://doi.org/10.1109/ JIOT.2019.2952141

United Nations. (n.d.a). Transforming our world: the 2030 Agenda for Sustainable Development. Department of Economic and Social Affairs. Sustainable Development. https:// sustainabledevelopment.un.org/post2015/transformingourworld

United Nations. (n.d.b). The 17 goals. Department of Economic and Social Affairs. Sustainable Development. https://sdgs.un.org/goals

Viriyasitavat, W., Da Xu, L., Bi, Z., \& Hoonsopon, D. (2019). Blockchain Technology for Applications in Internet of Things - Mapping From System Design Perspective. IEEE Internet of Things Fournal, 6(5), 8155-8168. https://ieeexplore.ieee.org/ document/8752029 
